\title{
Revalorización de las papas nativas de Bolivia (Solanum tuberosum L.) como fuente de hierro y zinc
} Revaluation of landraces potatoes from Bolivia (Solanum tuberosum $\mathbf{L}$.) as a source of iron and zinc

\author{
Julio Gabriel ${ }^{*}$, Rubén Botello ${ }^{1}$, José Luis Casazola ${ }^{2}$, Ricardo Vera ${ }^{3}$, Félix Rodríguez $^{1}$, Ada Angulo ${ }^{1}$
}

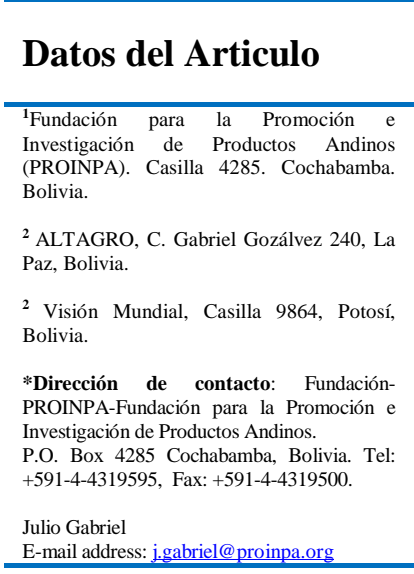

Palabras clave:

Especies,

micronutrientes,

cultivares,

biodiversidad,

nutrición.

\section{J. Selva Andina Res. Soc. 2014; 5(1):3-12.}

\section{Historial del artículo.}

Recibido mayo, 2014

Devuelto julio 2014

Aceptado julio, 2014

Disponible en línea, agosto, 2014.

\section{Editado por: \\ Selva Andina \\ Research Society}

\section{Key words:}

Species,

micronutrients

cultivars,

biodiversity,

nutrition

\section{Resumen}

Fueron recolectados en campo de agricultores de 19 comunidades del Norte Potosí, La Paz y Cochabamba, 164 cultivares nativos de papa en los años 2012 y 2013, con el objetivo de determinar el contenido de hierro y zinc en tubérculos. Los análisis del contenido de hierro y zinc fueron realizados en el laboratorio del Instituto de Tecnología de Alimentos (I.T.A.) de la Universidad Mayor Real y Pontificia de San Francisco Xavier de Chuquisaca, mediante la técnica de absorción atómica (AA). Los resultados mostraron que las especies con mayor contenido de hierro y zinc fueron Solanum $x$ ajanhuiri $(2 \mathrm{n}=2 \mathrm{x}=24)$, S. Solanum goniocalyx $(2 \mathrm{n}=2 \mathrm{x}=24)$ y S. stenotomum $(2 \mathrm{n}=2 \mathrm{x}=24)$. Sin embargo, el análisis de medias mostró que los cultivares con más alto contenido de hierro fueron Sak'ampaya (ADG) con $14.50 \mathrm{mg} \cdot \mathrm{kg}^{-1}$, Wila Surimana (STN) con $13.50 \mathrm{mg} \cdot \mathrm{kg}^{-1}$, Khati Señorita (STN) con $12.10 \mathrm{mg} \cdot \mathrm{kg}^{-1}$, Chilltu (STN) con $11.80 \mathrm{mg} \cdot \mathrm{kg}^{-1}$, Imilla Negra (ADG) con $10.90 \mathrm{mg} \cdot \mathrm{kg}^{-1}$, Yuraj Sak'ampaya (ADG) con $10.90 \mathrm{mg} \cdot \mathrm{kg}^{-1}$, Sak'ampaya (ADG) con $10.80 \mathrm{mg} . \mathrm{kg}^{-1}$ y Pinta Boca (STN) con $9.80 \mathrm{mgkg}^{-1}$. Para el contenido de zinc los contenido más altos fueron en los cultivares Yuraj Sak'ampaya (ADG) con $5.20 \mathrm{mg} \cdot \mathrm{kg}^{-1}$, Kellu Zapallo (GON) con 4.80 mg.kg-1 Ajawiri (AJH) con $9.00 \mathrm{mg} \cdot \mathrm{kg}^{-1}$, Pepino (STN) con $4.50 \mathrm{mg} \cdot \mathrm{kg}^{-1}$, Candelero (STN) con $4.50 \mathrm{mg} \cdot \mathrm{kg}^{-1} \mathrm{y}$ Zapallo (GON) con $4.40 \mathrm{mg} \cdot \mathrm{kg}^{-1}$. El análisis de correlación mediante la prueba de Pearson al $\operatorname{Pr}<0.05$ de probabilidad mostró una correlación moderada, positiva y notable $(\mathrm{r}=0.32)$ entre el contenido de hierro y zinc para todos los sitios donde se realizaron las colectas.

(C) 2014. Journal of the Selva Andina Research Society. Bolivia. Todos los derechos reservados.

\section{Abstract}

In 19 communities' farmer fields from Northern Potosi, La Paz and Cochabamba, 144 landraces potato were collected in the years 2012 and 2013, with the aim to determining the iron and zinc content in tubers. The content analysis of iron and zinc were performed in the Institute Food Technology (I.TA.) lab of the Royal and Pontifical San Francisco Xavier of Chuquisaca University, by using the technique of atomic absorption (AA). The results showed that species with higher content of iron and zinc were Solanum $x$ ajanhuiri $(2 \mathrm{n}=2 \mathrm{x}=24)$, S. Solanum goniocalyx $(2 \mathrm{n}=2 \mathrm{x}=24)$ and $S$. stenotomum $(2 \mathrm{n}=2 \mathrm{x}=24)$. However, the average analysis showed that cultivars with higher iron content were Sakampaya (ADG) con $14.50 \mathrm{mg} \cdot \mathrm{kg}^{-1}$, Wila Surimana (STN) with $13.50 \mathrm{mg} \cdot \mathrm{kg}^{-1}$, Khati Señorita (STN) with 12.10 mg.kg ${ }^{-1}$, Chilltu (STN) with $11.80 \mathrm{mg} \cdot \mathrm{kg}^{-1}$, Imilla Negra (ADG) with $10.90 \mathrm{mg} \cdot \mathrm{kg}^{-1}$, Yuraj Sak'ampaya (ADG) with $10.90 \mathrm{mg} \cdot \mathrm{kg}^{-1}$, Sak' ampaya (ADG) with $10.80 \mathrm{mg} \cdot \mathrm{kg}^{-1}$ and Pinta Boca (STN) with $9.80 \mathrm{mg} \cdot \mathrm{kg}^{-1}$. For zinc content, the highest content were in cultivars Yuraj Sak'ampaya (ADG) with $5.20 \mathrm{mg}^{\mathrm{kg}}{ }^{-1}$, Kellu Zapallo (GON) with 4.80 mg.kg-1 Ajawiri (AJH) with $9.00 \mathrm{mg} \cdot \mathrm{kg}^{-1}$, Pepino (STN) with $4.50 \mathrm{mg} \cdot \mathrm{kg}^{-1}$, Candelero (STN) with $4.50 \mathrm{mg} \cdot \mathrm{kg}^{-1}$ and Zapallo (GON) with $4.40 \mathrm{mg} \cdot \mathrm{kg}^{-1}$. Correlation analysis using Pearson's test at $\operatorname{Pr}<0.05$ probability showed a moderate correlation, positive and notable $(r=0.32)$ between the iron and zinc content for all sites where collections were made. 


\section{Introducción}

Bolivia forma parte del genocentro andino de origen y domesticación del cultivo de papa, que data de hace 8000 años a.C. (Hawkes 1990, Ochoa 2001). Hoy en día, en la región Andina de Bolivia aún existe una amplia diversidad de cultivares nativos concentrados en microcentros de biodiversidad, mantenidos por familias campesinas conservacionistas. Algunos de estos microcentros están localizados en la zona de Colomi ubicada en la provincia Chapare de Cochabamba (Terrazas \& Valdivia 1998, García \& Cadima 2003), en Llallagua, en el Norte de Potosí (Terrazas et al. 2008), y en el altiplano del Norte de La Paz (Iriarte et al. 2009).

Sin embargo, estudios recientes indican que en las zonas productoras de altura persisten condiciones de desnutrición crónica y que ocho de cada 10 niños (entre los 6 a 23 meses) padecen de anemia, considerada como el problema de deficiencia de macro y micro nutrientes con más prevalencia en el país (Mallea 2010).

La principal consecuencia de la deficiencia de hierro (Fe) es la anemia ferropénica. Sin embargo, también existen efectos adversos en el desarrollo psicomotor y cognitivo en menores de 2 años, la capacidad de aprendizaje, la conducta y la condición física. También existe mayor susceptibilidad a las infecciones (principalmente de tracto respiratorio), disminución de la velocidad de crecimiento y un incremento en la mortalidad infantil (Grandy et al. 2010).

La deficiencia de zinc ( $\mathrm{Zn}$ ) es frecuente en países en desarrollo (Brown et al. 2001) en los cuales hay baja ingesta de productos animales y alta ingesta de fitatos (Bhan et al. 2001). Un estudio realizado en escolares del área rural de Tanzania, Nepal e
India, mostró una prevalencia de deficiencia de $\mathrm{Zn}$ del $61 \%$ (punto de corte $<80 \mathrm{gdL}^{-1}$ ). Estudios revelan que niños con deficiencia moderada de $\mathrm{Zn}$ presentan retraso en el crecimiento lineal, al igual que efectos adversos en el desarrollo neuroconductual y desarrollo psicomotor. Asimismo, existe una disminución del apetito (debido a un efecto negativo en la capacidad para detectar el sabor de los alimentos) y de la respuesta inmune.

Las fuentes alimentarias de Fe y Zn son semejantes, por lo que sus deficiencias podrían esperarse que aparezcan de manera simultánea (Krebs \& Hambidge 1997). La diarrea, enfermedad responsable del $36 \%$ de las muertes en $<5$ años en Bolivia, aumenta la pérdida de zinc y hierro (Grandy et al. 2010).

En general, el niño o niña que ha sufrido desnutrición tendrá problemas en el desempeño escolar y de rendimiento cognitivo (Ordinola \& Fonceca 2012). Todo esto repercute posteriormente en bajos salarios y baja productividad (Burgos et al. 2007, Mallea 2010, Ordinola \& Fonceca 2012).

La papa es el cultivo que produce una gran cantidad de alimento nutricional por unidad de tiempo, agua y área y en climas muy adversos que cualquier otro cultivo mayor; hasta un $85 \%$ de la planta es comestible comparado con el de $50 \%$ de los cereales, convirtiéndola en una fuente muy importante para la alimentación. La papa contiene proteína de alto valor biológico, cantidades significativas de vitamina $\mathrm{C}$ (ácido ascórbico y dehidroascórbico) y otras vitaminas hidrosolubles: tiamina (vitamina B1) y vitamina B6. El contenido de minerales representa el $1.1 \%$ en los tubérculos de papa, siendo el potasio (K) el de mayor abundancia, el fósforo $(\mathrm{P})$, cloro $(\mathrm{Cl})$, azufre $(\mathrm{S})$, 
magnesio $(\mathrm{Mg})$, hierro $(\mathrm{Fe})$ y zinc $(\mathrm{Zn})$ están presentes en cantidades moderadas (Gabriel 2010). También proporciona oligoelementos esenciales como manganeso $(\mathrm{Mn})$, cromo $(\mathrm{Cr})$, selenio $(\mathrm{Se})$ y molibdeno (Mo). Además, su alto contenido de vitamina $\mathrm{C}$ mejora la absorción del Fe.

El contenido de proteína en la papa es análogo al de los cereales y es muy alto en comparación con otras raíces y tubérculos (Devaux et al. 2012).

La composición química de los tubérculos es variable y está controlada principalmente por factores genéticos dados por el cultivar, factores ambientales como: localidad, clima, suelo, agua, prácticas culturales y por la madurez de los tubérculos. La cocción y el almacenamiento también afectan la composición química de la papa y como consecuencia, su valor nutricional (Estrada 2000).

Investigaciones en el mejoramiento genético de la papa, están buscando desarrollar un valor agregado a la papa mediante la biofortificación (Burgos et al. 2007, Bonierbale et al. 2009), como una alternativa para mejorar el estado nutricional en las comunidades andinas, donde las personas no pueden pagar o acceder a alimentos enriquecidos o suplementos vitamínicos (Bouis \& Welch 2010, Devaux et al. 2012). Datos del Centro Internacional de la Papa (CIP) indican que la concentración media en los cultivares de papa es de $0.47 \mathrm{mg}$ de Fe por $100 \mathrm{~g}$ de peso fresco (4.7 $\mathrm{mg} . \mathrm{kg}^{-1}$ ) y $0.35 \mathrm{mg}$ de $\mathrm{Zn}$ por $100 \mathrm{~g}$ de peso fresco (3.5 mg. $\mathrm{kg}^{-1}$ ). La papa contribuye con $2.6 \%$ y 3.2 $\%$ de los requerimientos dietéticos diarios de Fe y Zn (Ortiz 2010). Varias investigaciones indican que la papa y particularmente los cultivares nativos, pueden ser una fuente complementaria de $\mathrm{Fe}$ y Zn (Ordinola \& Fonceca 2012).
Por lo mencionado, la presente investigación tuvo como objetivo determinar el contenido de Fe y $\mathrm{Zn}$ en tubérculos de cultivares nativos de papa de seis especies diferentes.

\section{Materiales y métodos}

Material vegetal. En los años 2012 y 2013 fueron colectados en 19 comunidades del Norte de Potosí, La Paz y Cochabamba 164 cultivares nativos, de los cuales 88 pertenecían a Solanum andigena (ADG), 8 a $S$. x ajanhuiri (AJH), 4 a $S$. goniocalyx (GON), 6 a $S$. x juzepczukii (JUZ), 57 a $S$. stenotomum (STN), uno a S. x curtilobum (CUR).

Zonas de recolección. La Figura 1, muestra los sitios de recolección de los cultivares de papa nativa.

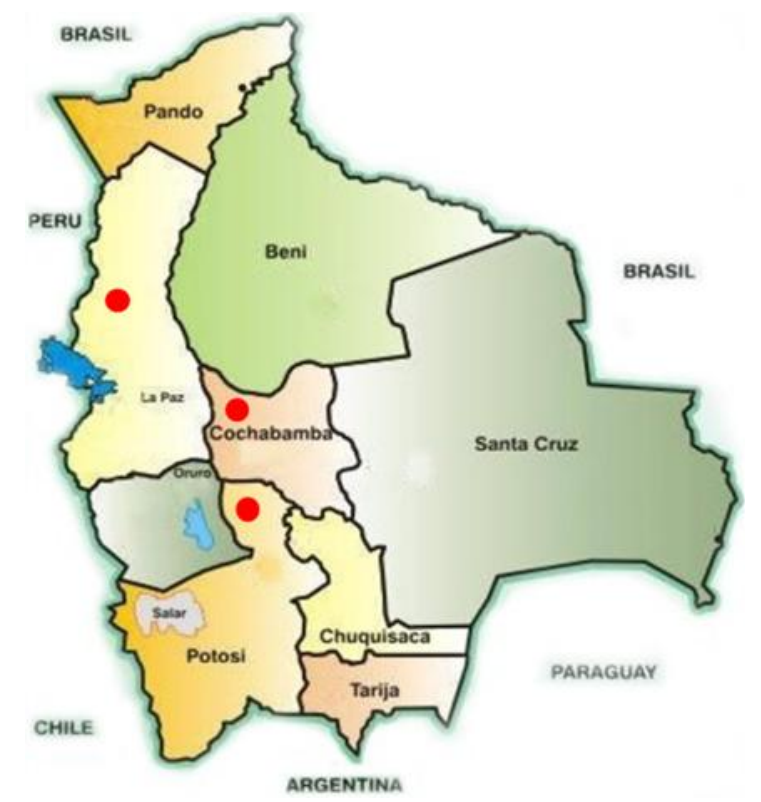

Figura 1. Mapa de Bolivia, donde se muestra los sitios de recolección de las papas nativas.

Tres comunidades se ubican en el departamento de Cochabamba a $17^{\circ} 23^{\prime}$ latitud sur y $66^{\circ} 09^{\prime}$ longitud oeste, entre 2600 a $4200 \mathrm{msnm}$, con tempe- 
ratura media de $15^{\circ} \mathrm{C}$, una precipitación media de $700 \mathrm{~mm}$ año ${ }^{-1}$ y HR de $80 \%$.

Doce comunidades del Norte de Potosí están en el departamento del mismo nombre a $18^{\circ} 26^{\prime}$ de latitud sur y $66^{\circ} 34^{\prime}$ longitud oeste, entre 3800 a $4017 \mathrm{msnm}$, con temperatura media de $8^{\circ} \mathrm{C}$, una precipitación media entre 300 a $400 \mathrm{~mm}^{2}$ año ${ }^{-1} \mathrm{y}$ HR de $40 \%$.

Cuatro comunidades de La Paz están ubicadas en la zona del Lago Titicaca en la provincia Franz Tamayo a $14^{\circ} 43^{\prime}$ de latitud Sud y $68^{\circ} 35^{\prime}$ de longitud Oeste, a una altitud de $3.383 \mathrm{msnm}$, con temperatura media de $12^{\circ} \mathrm{C}$, precipitación media de 500 mm.año ${ }^{-1}$ y HR de $60 \%$.

Recolección y envío de muestras. En la cosecha se recolectaron $1000 \mathrm{~g}$ de tubérculos de papa de cada variedad en campo de agricultores, estas fueron guardadas en bolsas de papel madera de 30 x 40 $\mathrm{cm}$ y etiquetadas cuidadosamente para conservar su identidad y evitar mezclas. Luego los tubérculos más sanos fueron re-seleccionados hasta lograr $500 \mathrm{~g}$ de muestra que fueron guardadas en bolsas de papel madera nuevos de $30 \times 40 \mathrm{~cm}$, las mismas que luego fueron enviados en cajas de cartón al laboratorio del Instituto de Tecnología de Alimentos (I.T.A.) de la Universidad Mayor Real y Pontificia de San Francisco Xavier de Chuquisaca (www.usfx.edu.bo/paginas/ita/) para el análisis del contenido de Hierro (Fe) y Zinc (Zn).

Análisis de minerales. Para determinar el contenido de $\mathrm{Fe}$ y $\mathrm{Zn}$ el I.T.A. utilizó la espectroscopia de Absorción Atómica (AA). La AA es una de las principales técnicas utilizadas en la cuantificación del contenido de minerales en muestras orgánicas. Ésta técnica espectroscópica tiene como fundamento la absorción de radiación de una longitud de onda determinada por parte de átomos libres, por lo tanto existe la necesidad de liberar el analito de la matriz que lo contiene dentro de un medio gaseoso, en el cual los átomos individuales están separados unos de los otros. Consecuentemente, el primer paso en todos los procedimientos espectroscópicos es la atomización, un proceso en el cual la muestra es volatilizada y descompuesta para producir un gas atómico. Esta radiación es absorbida selectivamente por átomos que tengan niveles energéticos cuya diferencia en energía corresponda a la energía de los fotones incidentes (Herrero \& Vigil 2003).

Condiciones para el análisis de muestra. Las condiciones ambientales de temperatura en los análisis estuvieron entre 16 a $18{ }^{\circ} \mathrm{C}$ y entre 26 a $51 \%$ de humedad relativa. El análisis fue realizado en base húmeda en mg.100g $\mathrm{g}^{-1}$ tanto para hierro como para zinc. Los datos fueron transformados posteriormente a mg. $\mathrm{kg}^{-1}$.

Análisis estadísticos. Para evaluar la variabilidad de las concentraciones de $\mathrm{Fe}$ y $\mathrm{Zn}$ entre las especies, se realizó un análisis de varianza (ANVA), considerando un diseño completamente aleatorizado. Las medias se compararon mediante la prueba múltiple de Duncan $(\operatorname{Pr}<0.05)$. También se determinó el intervalo de confianza al 95\% (IC) para las medias del contenido de Fe y Zn. Finalmente se realizó un análisis de correlación mediante la prueba de Pearson entre las concentraciones de Fe y $\mathrm{Zn}$ de todas las localidades. Todas las estadística de prueba se realizaron con el SAS versión 9.2 (SAS 2004). 


\section{Resultados}

La Tabla 1 muestra el ANVA realizado para el contenido de $\mathrm{Fe}$ y $\mathrm{Zn}$ (C.V. de $30 \%$ y $20 \%$ respectivamente), encontrándose diferencias significativas $(\operatorname{Pr}<0.05)$ entre especies para el contenido de $\mathrm{Zn}$ y no así para Fe. Esto indica que aparentemente el contenido de $\mathrm{Fe}$ no varía sustancialmente entre especies en las zonas recolectadas.

Tabla 1. Análisis de varianza del contenido de Fe y Zn en tubérculos de papa nativa.

\begin{tabular}{lccc}
\hline FV & Gl & Fe $\left(\mathbf{m g . k g} \mathbf{~}^{-\mathbf{1}}\right)$ & Zn $\left(\mathbf{m g . k g} \mathbf{k g}^{\mathbf{- 1}}\right)$ \\
\hline Total & 143 & & \\
Especie & 5 & $3.41 \mathrm{~ns}$ & $1.08^{*}$ \\
Error & 138 & 4.68 & 0.39 \\
C.V. $(\%)$ & & 30.60 & 20.70 \\
\hline
\end{tabular}

$*=$ Significativo al $\mathrm{p}<0.05$ de probabilidad

Sin embargo, la comparación de medias para el contenido de $\mathrm{Fe}\left(\mathrm{mg} \cdot \mathrm{kg}^{-1}\right)$ entre las especies de papa nativa mediante la prueba múltiple de Duncan $\quad(\operatorname{Pr}<0.05) \quad$ (Figura 2) mostró que la especie CUR posee menor contenido de $\mathrm{Fe}$ con respecto a las especies AJH, GON y STN, que no mostraron diferencias notables entre sí. Por otra parte el contenido de Fe de la especie ADG y JUZ es igual a CUR y al resto de las especies.

Se debe mencionar que los cultivares con más alto contenido de Fe fueron los cultivares Sak'ampaya (ADG) con $14.50 \mathrm{mg} \cdot \mathrm{kg}^{-1}$, Wila Surimana (STN) con $13.50 \mathrm{mg} \cdot \mathrm{kg}^{-1}$, Khati Señorita (STN) con $12.10 \mathrm{mg} \cdot \mathrm{kg}^{-1}$, Chilltu (STN) con $11.80 \mathrm{mg} \cdot \mathrm{kg}^{-1}$, Imilla Negra (ADG) con $10.90 \mathrm{mg} \cdot \mathrm{kg}^{-1}$, Yuraj Sak'ampaya (ADG) con $10.90 \quad \mathrm{mg} \cdot \mathrm{kg}^{-1}$, Sak'ampaya (ADG) con 10.80 mg.kg- y Pinta Boca (STN) con $9.80 \mathrm{mg} \cdot \mathrm{kg}^{-1}$ (Figura 3).
Figura 2. Contendio de $\mathrm{Fe}\left(\mathrm{mg} \cdot \mathrm{kg}^{-1}\right)$ en seis especies nativas de papa boliviana.

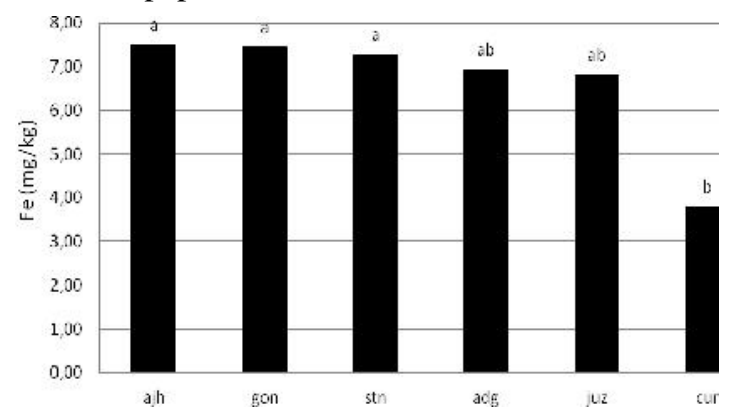

En referencia al análisis de contenido de $\mathrm{Zn}$ $\left(\mathrm{mg} . \mathrm{kg}^{-1}\right)$, la comparación de medias (Figura 4) mostró que la especie GON fue superior en contenido de $\mathrm{Zn}$ a CUR $(\operatorname{Pr}<0.05)$; por otra parte, las especies STN, JUZ, ADG y AJH no mostraron diferencias notables entre sí.

Figura 3. Cultivares nativos de papa con alto contenido de hierro.

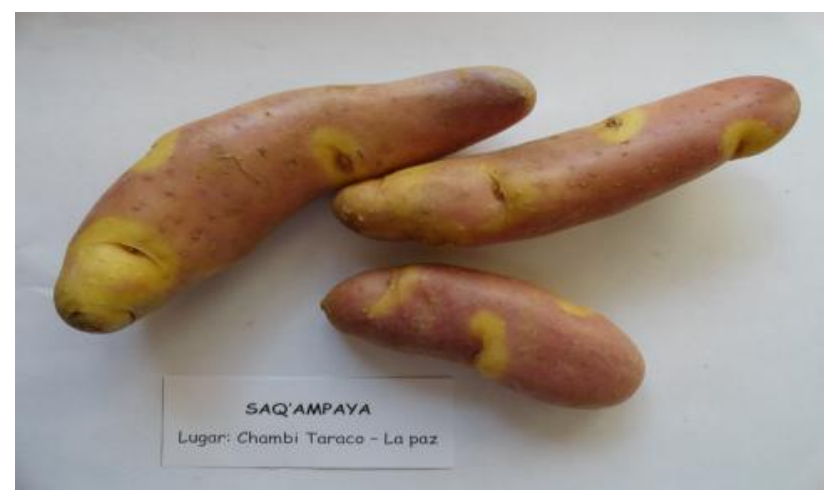

a) Sak'ampaya (ADG-Tetraploide)

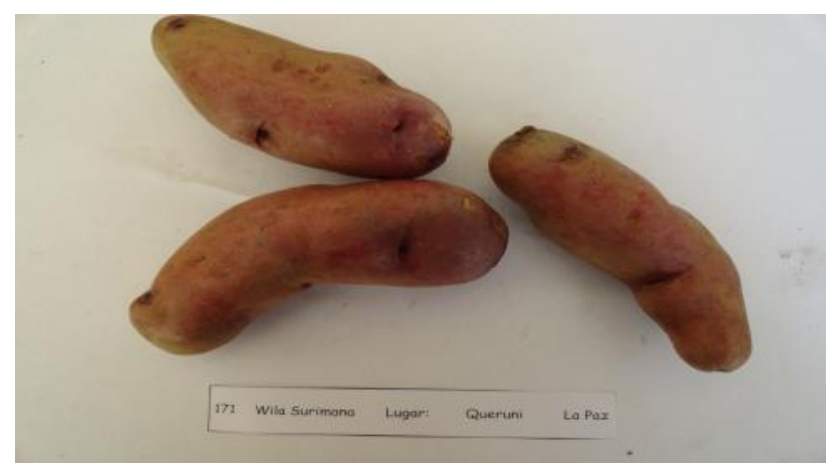

b) Wila surimana (STN-Diploide) 


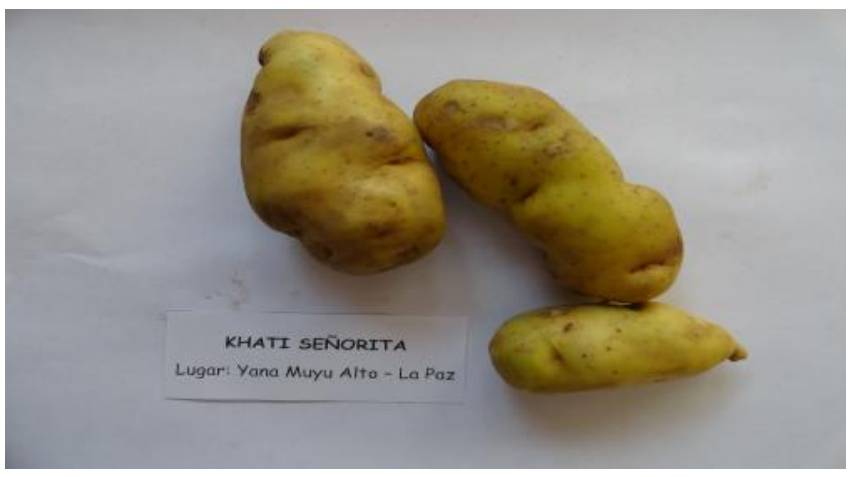

c) Khati Señorita (STN-diploide)

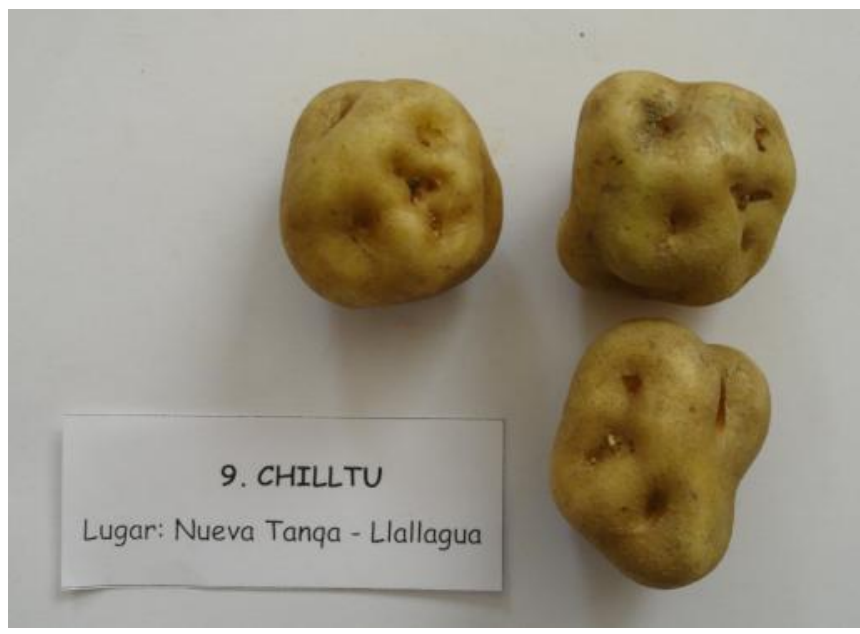

d) Chilltu (STN-diploide)

Se debe indicar que los contenido más altos de $\mathrm{Zn}$ fueron para los cultivares Yuraj Sak'ampaya (ADG) con $5.20 \mathrm{mg} \cdot \mathrm{kg}^{-1}$, Kellu Zapallo (GON) con 4.80 mg. $\mathrm{kg}^{-1}$, Ajawiri (AJH) con $4.50 \mathrm{mg} \cdot \mathrm{kg}^{-1}$, Pepino (STN) con $4.50 \mathrm{mg} \cdot \mathrm{kg}^{-1}$, Candelero (STN) con 4.50 mg. $\mathrm{kg}^{-1}$ y Zapallo (GON) con 4.40 mg.kg-1 (Figura 5).

Solamente el cultivar Yuraj Sak'ampaya obtuvo los más altos contenidos tanto para Fe como para $\mathrm{Zn}$.

El intervalo de confianza al 95\% (Tabla 2), mostró que la desviación es mayor para el contenido de Fe que para el de $\mathrm{Zn}$ y que el rango de variación para el contenido de Fe está entre 2.3 a 13.5 mg. $\mathrm{kg}^{-1}$ como valores mínimos y máximos respectivamente. En cambio en el $\mathrm{Zn}$ el rango de contenido varía entre 0.9 a 5.2 mg.kg-1 como valores mínimos y máximos respectivamente.

Tabla 2. Intervalos de confianza al $95 \%$ del contenido de Fe y $\mathrm{Zn}$ en seis especies nativas de papa boliviana.

\begin{tabular}{ccccc}
\hline $\begin{array}{c}\text { Micro } \\
\text { nutrientes }\end{array}$ & $\mathbf{N}$ & Desviación & Promedio & I.C. (95\%) \\
\cline { 3 - 5 } & & & & mg.kg $^{-1}$ \\
\hline $\mathrm{Fe}$ & 164 & 2.15 & 7.06 & $2.3-14.5$ \\
$\mathrm{Zn}$ & 164 & 0.64 & 3.02 & $0.9-5.2$ \\
\hline
\end{tabular}

Figura 4. Contenido de $\mathrm{Zn}\left(\mathrm{mg} \cdot \mathrm{kg}^{-1}\right)$ en seis especies nativas de papa boliviana.

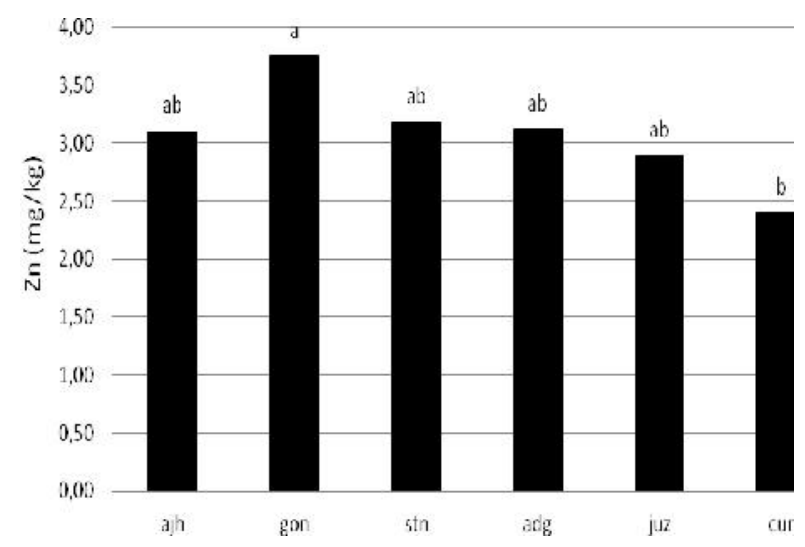

El análisis de correlación mediante la prueba de Pearson $(\operatorname{Pr}<0.05)$ mostró una correlación moderada y positiva $(r=0.32)$ (Figura 6$)$ entre el contenido de $\mathrm{Fe}$ y $\mathrm{Zn}$ para todas las localidades. 
Figura 5. Cultivares nativos de papa con alto contenido de zinc.

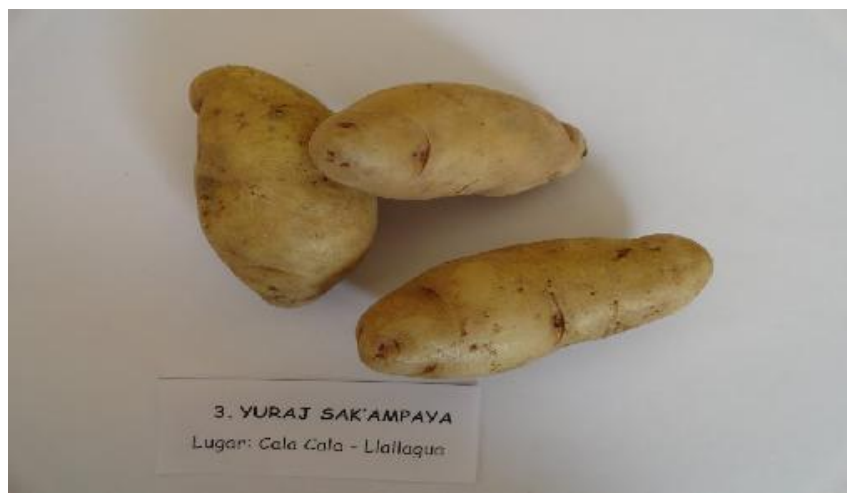

a) Yuraj Sak'ampaya (ADG-Tetraploide)

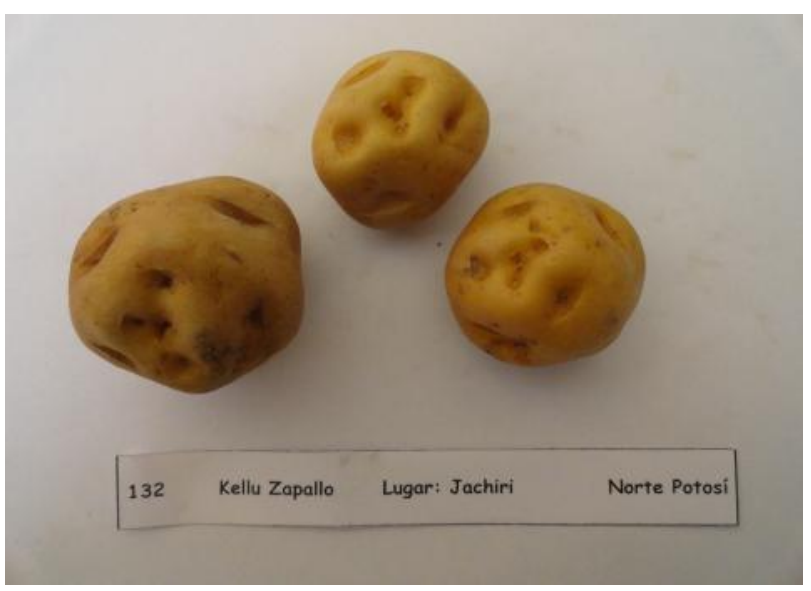

b) Kellu Zapallo (GON-Diploide)

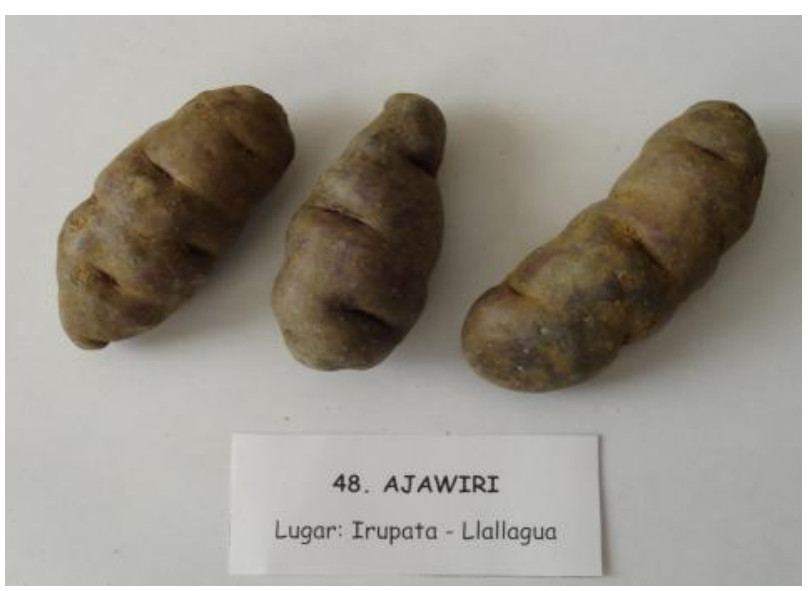

c) Ajawiri (AJH-diploide)

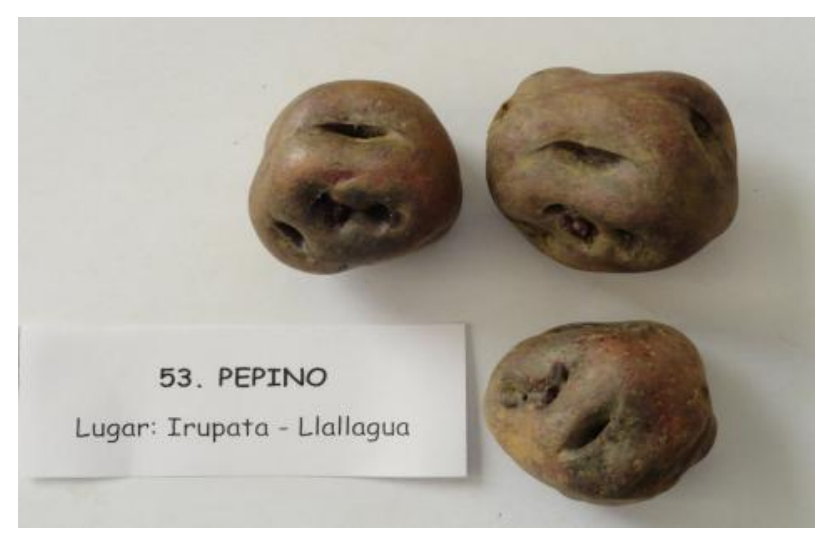

d) Pepino (STN-diploide)

Figura 6. Correlación del contenido de Fe $\left(\mathrm{mg} \cdot \mathrm{kg}^{-1}\right)$ y $\mathrm{Zn}\left(\mathrm{mg} \cdot \mathrm{kg}^{-1}\right)$ de seis especies nativas de papa boliviana, en todas las localidades de recolección.

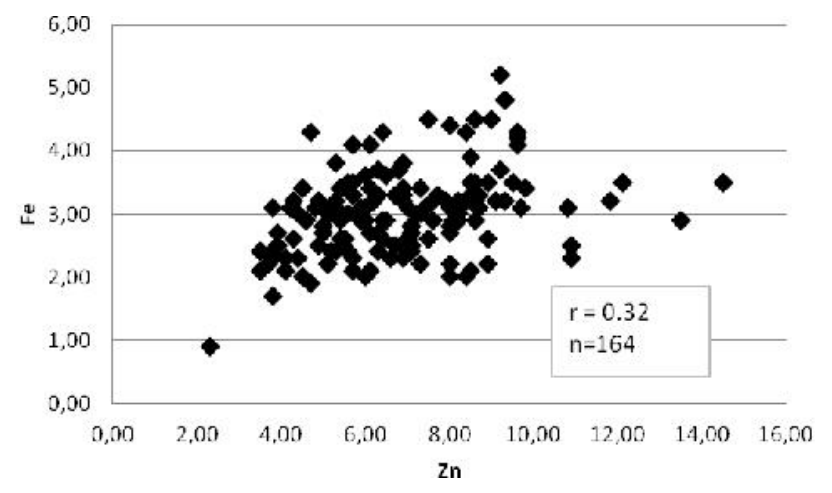

\section{Discusión}

Niveles significativos de $\mathrm{Fe}$ y $\mathrm{Zn}$ y moderada disponibilidad de $\mathrm{Fe}$ fueron reportados en papa (True et al. 1978, Casañas et al. 2003, Fair Weather-Tait 2003) y en Perú se reportó una concentración media de 4.7 mg. $\mathrm{kg}^{-1}$ de Fe y 3.5 mg.kg-1 de Zn en cultivares de papa (Ortiz 2010).

En nuestro estudio, hemos encontrado concentraciones medias de Fe más altas que las reportadas en cinco de las seis especies evaluadas (excepto CUR). 
Para Zn por otra parte, se encontró una concentración media superior a la reportada solamente en la especie GON. Estos niveles de concentración pueden aún incrementarse más a través de la mejora genética, como lo indican Burgos et al. (2007).

Los mismos autores reportaron contenidos superiores de $8.6 \mathrm{mg} . \mathrm{kg}^{-1}$ de Fe y de $5.3 \mathrm{mg} . \mathrm{kg}^{-1}$ de $\mathrm{Zn}$ en germoplasma de papa nativa. Los mayores contenidos de $\mathrm{Fe}$ reportados en nuestro estudio fueron superiores $\left(>9.8 \mathrm{mgkg}^{-1}\right)$.

Debemos mencionar que hemos observado niveles inferiores aunque no significativos de $\mathrm{Fe}$ y $\mathrm{Zn}$ en ADG, como es el caso del cultivar Waych'a la cual es una de las más cultivadas del país (Zeballos et al. 2009), y que mostró un nivel promedio de Fe de 6.5 mg. $\mathrm{kg}^{-1}$ y de $\mathrm{Zn}$ de 2.5 $\mathrm{mg} \cdot \mathrm{kg}^{-1}$, en ADG similares resultados fueron encontrados por López et al. (2009).

En este mismo estudio (López et al 2009) reportaron que $S$. x chaucha $(2 \mathrm{n}=3 \mathrm{x}=36)$ mostró los niveles más altos de Fe. No hay antecedentes sobre el contenido de Fe y Zn en especies amargas como CUR, la cual mostró bajos niveles de Fe y $\mathrm{Zn}$.

Con los niveles observados en este estudio, las mujeres y niños de las comunidades donde se realizaron las colectas podrían incrementar su ingesta de $\mathrm{Fe}$ significativamente a través del consumo de un cultivar con alto contenido de Fe. Estudios anteriores sugirieron que debido a los niveles altos de ácido ascórbico (promotor de la absorción de $\mathrm{Fe}$ ) y niveles bajos de ácido fítico (inhibidor de la absorción de hierro) en papa, la bio-disponibilidad de $\mathrm{Fe}$ es moderadamente alta (Fair Weather-Tait 2003). Sin embargo, para que estos cultivares sean utilizados a gran escala se requiere desarrollar programas más organizados de producción de semilla de calidad.

El nivel de concentración de Fe encontrado en las especies AJH, GON y STN podría suplir el $25 \%$ del consumo diario de Fe recomendado para niños de entre 1 a 3 años (6 mg.día ${ }^{-1}$ ) y el $21 \%$ del consumo diario de $\mathrm{Fe}$ recomendado para mujeres en edad fértil (29 mg.día ${ }^{-1}$ (FAO 2001), considerando una ingesta diaria promedio de papa de 200 y 800 g para niños y mujeres respectivamente. Asimismo, el cultivar Ajawiri; podría suplir un 36 y un $30 \%$ de las necesidades diarias de Fe para niños y mujeres respectivamente (Burgos et al. 2007).

Para el Zn, el consumo diario de AJH, GON y STN supliría el 15 y $18 \%$ respectivamente del consumo diario recomendado de $\mathrm{Zn}$ para niños entre 1 a 3 años (4.1 mg.día ${ }^{-1}$ ) y el 51 y $61 \%$ del consumo diario recomendado para mujeres en edad fértil (4.9 mg.día ${ }^{-1}$ ) (FAO 2001). S. x ajanhuri por su parte, supliría el 25 y $85 \%$ de las necesidades diarias de $\mathrm{Zn}$ de niños y mujeres respectivamente (Burgos et al. 2007).

$S$. x ajanhuri y los demás cultivares de estas especies podrían ser utilizados como una fuente valiosa para mejorar los niveles de Fe y Zn para la dieta o la generación de nuevos cultivares diploi$\operatorname{des}(2 \mathrm{n}=2 \mathrm{x}=24)$ y tetraploides $(2 \mathrm{n}=4 \mathrm{x}=48)$ biofortificados, cuyo alto contenido de $\mathrm{Fe}$ y $\mathrm{Zn}$ puede transferirse a papas comerciales (Haynes \& Abdul-Bakj 1993, Mendoza 1989)

Burgos et al. (2007) reportaron correlaciones positivas entre 0.52 y 0.54 para dos zonas altas productoras de papa nativa. Aunque la correlación que hemos encontrado fue relativamente más baja que las reportadas, sugiere que se podrían realizar selecciones simultáneas de los mejores niveles de ambos microelementos en este valioso germoplas- 
ma, pero se debe tomar en cuenta el contenido de materia seca, como sugieren Burgos et al. (2007).

\section{Conflictos de intereses}

Los autores declaran que no existen conflictos de interés.

\section{Agradecimientos}

Esta investigación se realizó con ayuda financiera de la Comisión de la Unión Europea a través del FIDA y el apoyo técnico del CIP al Proyecto IssAndes. Las opiniones expresadas en el mismo no reflejan necesariamente la opinión oficial de la Comisión de la Unión Europea.

\section{Literatura citada}

Bhan MK, Sommerfelt H., Strand T. Micronutrient deficiency in children. Br J Nutr. 85, Suppl. 2001; 2: S199- S203.

Bonierbale M, Gruneberg W, Amoros W, Burgos G, Salas E, Porras E, et al. Total and individual carotenoid profiles in Solanum phureja cultivated potatoes: II. Development and application of near-infrared reflectance spectroscopy (NIRS) calibrations for germ-plasm characterrization. J Food Compos Anal. 2009; 22: 509516.

Bouis HE, Welch RM. Biofortification - A Sustainable Agricultural Strategy for Reducing Micronutrient Malnutrition in the Global South. Crop Sci. 2010; 50:20-32.

Brown K, Wuehler SE, Peerson JM. The importance of zinc in human nutrition and estimation of the global prevalence of zinc deficiency. Food Nutr Bull. 2001; 22: 113-25.
Burgos G, Amoros W, Morote M, Stangoulis J, Bonierbale M. Iron and zinc concentration of native Andean potato cultivars from a human nutrition perspective. J. Sci. Fd. Agric. 2007; 87:668-675.

Casañas R, Suarez P, Rodríguez E, Darias J, Díaz C. Mineral concentration in cultivars of potatoes. Food Chem. 2003; 83:247-253.

Devaux A, Andrade-Piedra J, Ordinola M, Velasco C, Hareau G, López G, et al. La Papa y la seguridad alimentaria en la región andina: situación actual y desafíos para la innovación. Revista COSUDE. 2012; 46-49.

Estrada N. La Biodiversidad en el Mejoramiento Genético de la papa. Bill Hardy, Emma Martinez (Ed.) La Paz, Bolivia. 2000; 372 pp.

Fair Weather-Tait S. Studies on the availability of iron in potatoes. Br J Nutr. 2003; 50:15-23.

FAO. Human Vitamin and Mineral Requirements. Report of a joint FAO/WHO expert consultation Bangkok, Thailand. 2001; 281 pp.

Gabriel J. Documento marco: Estrategias y perspectivas del mejoramiento genético de papa (Solanum tuberosum L.) en Bolivia. Fundación PROINPA, Cochabamba, Bolivia. 2010; 60 p.

Grandy G, Weisstaub G, López de Romaña D. Deficiencia de hierro y zinc en niños. Rev Soc Bol Ped. 2010; 49 (1): 25-31.

García W, Cadima X. (Ed) Manejo sostenible de la agrobiodiversidad de tubérculos andinos: Sínte-sis de investigaciones y experiencias en Boli-via. Conservación y uso de la biodiversidad de raíces y tubérculos andinas: Una década de investigación para el desarrollo (1993-2003). 1. Fundación para la Promoción y la Investiga-ción de Productos Andinos (PROINPA), Alcal-día de Colomi, Centro Internacional de la Papa (ClP) Agencia Suiza 
para el Desarrollo y la Cooperación (COSUDE). Cochabamba, Boli-via. 2003; 208 pp.

Hawkes JG. The potato : evolution, biodiversity and genetic resources. Belhaven, London, UK. 1990; 259 pp.

Haynes KG, Abdul-Bakl AA. Male fertility of derived teraploids of Solanum tuberosum from groups tuberosum x Phureja-stenotomum. AM Potato J. 1993; 70:814-815.

Herrero E, Vigil A. Metodología recomendada para la medición del contenido de zinc en especímenes biológicos. Química Clínica. 2003; 22 (1) $13-18$.

Iriarte V, Condori B, Parapo D, Acuña D. Catálogo etnobotánico de papas nativas del Altiplano Norte de La Paz-Bolivia. Cochabamba, Bolivia. 2009; 142 pp.

Krebs N, Hambidge M. Trace elements in human nutriation. Page 91-114 in Nutrition in Pediatrics: Basic Science and clinical applications. Edited by Walker WA, Watkins JB. BC Decker Inc. Publisher (London). 1997.

López R, Barandalla L, Ritter E, Hasse UN, Ruiz de Galarreta JI. Evaluación del valor nutricional de germoplasma nativo de patata para su incorporación en programas de mejora genética. Rev latinoam papa. 2009; 15 (1):55-57.

Mallea I. Situación actual y prioridades básicas de la seguridad alimentaria nutricional en Bolivia. CienciAgro. 2010; 2 (1): 237-252.

Mendoza HA. Population breeding as a tool for germplasm enhancement. AM Potato J. 1989; 66:639-653.

Ochoa C. Las papas de sudamérica : bolivia. Plural Editores/CID. 2001; 535 pp.
Ordinola M., Fonceca C. Papas nativas: Aportes para la seguridad alimentaria, nutrición y generación de ingresos en zonas altas del Perú. Páginas 3 en XXV Congreso de la ALAP, Uberlandia-MG, Brasil. 2012.

Ortiz R (Ed.). La Biofortificación de los cultivos para combatir la anemia y las deficiencias de micronutrientes en el Perú. Programa Mundial de Alimentos (PMA), Lima, Perú. 2010; 39 pp.

SAS Institute Inc. SAS/STAT Users Guide, Version 9.2, Fourth Edition, Vol. 2, Cary, N.C. 2004.

Terrazas F, Cadima X, García R, Zeballos J. Catálogo etnobotánico de papas nativas. Tradición y cultura de los Ayllus del Norte Potosí y Oruro. Ricerca \& Cooperazione, Unión Europea, Centro de Apoyo a Desarrollo, GTZ, Fundación PROINPA, MDRyMA. Cochabamba - Bolivia. 2008; 189 pp.

Terrazas F, Valdivia G. Spatial dynamics of in situ conservation: handling the genetic diversity of Andean tubers in mosaic systems. Genet Resour Newsl. 1998; 114:9-15.

True RH, Hogan JM, Augustin J, Johnson SR, Teitzel C, Toma RB, et al. Mineral composition of freshly harvested potatoes. AM Potato J. 1978; 55: 511-519.

Zeballos H, Balderrama F, Condori B, Blajos J. Economía de la papa en Bolivia (1998-2007). Fundación PROINPA. Cochabamba, Bolivia. 2009; 129 pp. 